\title{
INTRODUCTION \\ Strategy and Outline of the Primer
}

$\mathbf{T}$

HIS book provides the scientific basis required to assess what changes to the climate might plausibly come about in the future and what might cause these changes to occur. It provides the basics of physical climatology set out with the objective of developing an understanding of how human activity might lead to changes in climate and what form those changes might take. As such, it does not attempt to cover all aspects of climatology. One central theme is to elaborate the origin of the inherent and irreducible uncertainty in predictions of possible climate futures.

This text emphasizes physical aspects of the climate systemwinds, temperature, ocean currents, atmospheric circulation, and rainfall—both in building an understanding of the mechanics of the climate system and in describing drivers of change to the system and potential consequences, such as sea level rise and hurricane dynamics. Aspects of the climate system and climate change that depend upon and may influence ecological systems are dealt with in other books in the Columbia University Earth Institute Sustainablity Primers series.

Most natural science fields, including climate science, make gains through a process of successive approximations. The first 
approximation describes the most fundamental aspect of the system under study. For example, as a first approximation, we know that atoms are made of neutrons, protons, and electrons. Although we now know that atoms are much more complex than this, with scores of subatomic particles, that knowledge has not made the initial description incorrect-there are still neutrons, protons, and electrons. However, newer and progressively more refined approximations can be made that lead to a more complete description of the atom. No physicist would suggest, even with verification of the Higgs boson, that atoms are now fully described. In the same way, no climate scientist would suggest that the climate system has been fully described and understood. It is, however, known at a level sufficient to say how human activity can influence climate.

Bertrand Russell expressed this idea succinctly: "Science does not aim at establishing immutable truths and eternal dogmas; its aim is to approach the truth by successive approximations, without claiming that at any stage final and complete accuracy has been achieved." The structure of this text follows Russell's theme of successive approximations in describing our understanding of the climate system. In taking this approach, I also follow a narrative arc that takes us from some of the earliest descriptions of the climate system to the present day, where much of the focus is on climate prediction. I also discuss some of the consequences of climate change in the context of sustainable development.

In the first approximation, we imagine Earth to be essentially static. There are no oceans and no vegetation, and the only feature of the atmosphere is clouds. From this starting point, I develop a theory that explains why Earth's average temperature is around $16^{\circ} \mathrm{C}$. This leads directly to the greenhouse effect, using basic elements of well-established atomic physics, and to the inference that human activity can indeed alter climate through modification of the atmosphere's chemical composition. 
The second level of approximation acknowledges that Earth is a spheroid rotating on its axis, and that the temperature varies with location on the planet. From these facts, I develop an understanding of wind patterns, atmospheric and oceanic circulation, plus rainfall patterns and their variations. Developing this second-order approximation in no way invalidates conclusions drawn from the first level of approximation, but it adds features and details of the climate system not available in the first approximation. The second approximation draws on classical Newtonian mechanics.

The next level of refinement, the third approximation, examines several dynamic features of climate, including the glacial/ interglacial cycles, the E1 Niño Southern Oscillation, and other features of Earth's natural dynamics. Here we need to include some aspects of the behavior of the ocean and its coupled interaction with the atmosphere. This chapter includes a discussion of the consequences of oscillating climate states on malaria prevalence and crop production in poor countries. A discussion of the issues around prediction of dynamic systems is also included.

Through these steps, a sufficient understanding of the climate system is built so we can analyze the critical question of Earth's future climate through use of climate model projections. In particular, I examine the reasons for uncertainties when predicting the climate's distant future.

I conclude with three examples of the consequences of climate warming that illustrate how particular aspects of Earth's system might be modified under climate change (through sea level rise and tropical cyclones) together with some comments on future climate variability and committed warming, which is the warming to be expected even were the concentration of greenhouse gases to stabilize. 
The text is derived from class notes prepared by the author for a course titled Science for Sustainable Development, cotaught with Professor Ruth DeFries to undergraduates at Columbia University. The class notes have proven useful for some master's and PhD students as well.

Material contained in boxes is more technical in nature and is parenthetical in form; it need not be read to understand the main body of the text. It is included for completeness for readers who have more preparation in the physical sciences and who may wish to have a greater level of detail on certain subjects.

A glossary contains terms that may be unfamiliar to readers new to the field. These terms, when first introduced in the text, appear in italics. Illustrations are taken from the published literature in most cases, and they generally began as color images that have been transformed to grayscale images, with varying levels of success. References to the original color images are provided in the captions to the figures. 


\section{CLIMATE CHANGE SCIENCE}


